\title{
Vocational and Educational Training in Family and Community Nursing as a European Priority: The ENhANCE Erasmus+ Project. Aspects for Mental Health Care
} \author{
Diab $^{12}$, \\ 1TEI of Thessaly, Greece \\ 2 ITD-CNR, Italy \\ $3 \mathrm{Si} 4 \mathrm{Life}$, Italy \\ 4 University of Genoa, Italy \\ 5 ALISA, Italy \\ 6 EUROCARERS, Belgium \\ 7 EASPD, Belgium \\ 8 University of Eastern Finland, Finland \\ 9 TEI of Crete, Greece \\ 10 ENE, Greece \\ 11 Future Balloons, Portugal \\ 12 AWV, Germany
}

Ioanna V. Papathanasiou ${ }^{1}$, Evangelos C. Fradelos ${ }^{1}$, Sofia Kastanidou ${ }^{1}$, Konstantinos Tsaras ${ }^{1}$, Francesca Pozzi ${ }^{2}$, Flavio Manganello ${ }^{2}$, Serena Alvino ${ }^{3}$, Loredana Sasso ${ }^{4}$, Annamaria Bagnasco ${ }^{4}$, Giuseppe Aleo ${ }^{4}$, Isabella Roba ${ }^{5}$, Stecy Yghemonos $^{6}$, Adriana Popa ${ }^{7}$, Hannele Turunen ${ }^{8}$, Christos Kleisiaris ${ }^{9}$, Eftychia Evangelidou ${ }^{10}$, Aris Daglas ${ }^{10}$, Clara Rodrigues $^{11}$, Lars Oertel ${ }^{12}$, Madeleine

\begin{abstract}
.
Family and Community Nurses play a vital role in many aspects of EU healthcare systems, as more and more patient are treated outside the traditional hospital settings. Family and Community Nurses provide nursing interventions and also educational and counselling services for patients and families, as well as for total communities. So, they should be trained properly to perform a variety of nursing procedures. EU and World Health Organization (WHO) reports identify the Family and Community Nurse (FCN) as a key-actor in the new Primary Health Care (PHC) model.
\end{abstract}

Concerning community mental health care, current studies' results have shown many benefits for individuals that are suffering from mental disorders and their families also, of the nursing care that is provided from Family and Community Nurses. According to these results the patient - centered, adequate and continuing nursing care is an essential condition for better health outcomes of chronic mental health disorders, can reduce relapses and hospitalizations, saving significant funds.

The ENhANCE Erasmus+ Project (https://www.enhance-fcn.eu/ ) targets to the existing mismatch between the skills currently offered by nurses working in Primary Health Care (PHC) and those actually demanded by both public health care institutions and private service providers when applying innovative healthcare models centered on PHC. The Alliance of the project is composed of 13 partners, from 6 different EU countries representing VET providers in the field of Nursing, Regulatory Bodies for FCN training, Professional Associations and Public and Private Employers.

To achieve the project objectives, the ENhANCE Alliance is carrying out a number of activities including:

- Definition and delivery of a Professional Profile for the Family and Community Nurse (FCN) which could be taken as a reference at European level for the Vocational Education and Training (VET).

- Design of a Curriculum for Family and Community Nurse (FCNs) which could play a reference role at European level.

- Development of specific guidelines/instructions supporting VET providers in the instantiation of the EU Curriculum into local curricula.

- Delivery of a training course for teachers and development of guidelines and methodological recommendations for them on how to effectively use and tailor the tools and methods proposed by the 
Papathanasiou1, Fradelos1, Kastanidou1, Tsaras1, Pozzi2, Manganello2, Alvino3, Sasso4, Bagnasco4, Aleo4,

Roba5, Yghemonos6, Popa7, Turunen8, Kleisiaris9,

Evangelidou10, Daglas10, Rodrigues11, Oertel12, Diab12

MHGCJ - 2018

project.

- Development of an innovative open online tool supporting case studies and best practice sharing for nurses

- Design of 3 national FCN curricula and implementation of 3 pilot courses.

- Development of Recommendations for efficient investments on FCN professionals both for public and for private employers.

- Evaluation and Quality assurance of the project results.

- Dissemination of the project outputs among different stakeholders.

- Exploitation of the results and assurance for project sustainability.

As for mental health aspects, the results from an e-Delphi study that was conducted under the ENhANCE project, and in which experts in FCN from EU countries participated in order key FCN competencies to be identified, have shown that 28 competencies are the core for delivery quality nursing care in the community. Many of those competencies are vital on providing community based mental health care, and will be mentioned. 